\title{
The Effect of Practice Modules Based Nursing Simulation Learning on Communication Ability and Learning Experience of Nursing Students
}

\section{임상 실무 기반 간호 시뮬레이션 교육이 간호학생의 의사소통 능력과 수업경험에 미치는 효과}

Eun Hui Seo ${ }^{1}$, Seon Il Park ${ }^{2}$

서은희 ${ }^{1}$, 박선일 ${ }^{2}$

${ }^{1}$ Professor, Department of Nursing, Chodang University, Korea, sillvia1004@cdu.ac.kr

${ }^{2}$ Professor, Departmen of Nursing, Kwangju Women`s University, Korea, psi2161@kwu.ac.kr

Corresponding author: Seon Il Park

\begin{abstract}
The purpose of study was to determine the effect of practice modules based nursing simulation learning in enhancing the nursing practice competency of nursing students and to investigate the effects on communication ability and learning experience of nursing students. This study utilized a nonequivalent pre-test posttest quasi-experimental design. A total of 254 nursing students participated in the study with 122 in the experimental group and 132 in the control group. The subjects of this study are third year students from the nursing department. Practice modules based nursing simulation was conducted for 15 weeks in 4 modules. The pre-post data collection of the experimental group was investigated for communication ability through questionnaires, and education experiences of the experimental group was investigated after the questionnaire. Data were analyzed using the SPSS 20.0 program with descriptive statistics. The outcome measurements were communication ability, education experience. The experiment group was more communication ability 164.5 points than the control group 156.8 points with the difference being significant $(\mathrm{t}=4.33, p<.001)$, simulation learning experience of the experimental group received nursing simulation learning was statistically significant $(\mathrm{t}=15.38, p<.001)$. Active in various nursing fields nursing simulation learning will be conducted, and simulations to get a basic nursing education from grade performance by conducting site clinical training seems to be able to promote.
\end{abstract}

Keywords: Simulation, Practice Modules, Communication Ability, Learning Experience

요약: 본 연구는 간호 실무 역량을 향상할 수 있는 임상 실무 기반 간호 시뮬레이션을 간호학 생에게 적용하여 의사소통 능력 및 수업 경험에 미치는 효과를 파악하고자 시도되었다. 연구 대상자는 총 254 명으로 실험군 122 명, 대조군 132 명이며 간호학과에 재학 중인 3 학년 간호 학생이다. 시뮬레이션 교육은 4 가지 모듈로 구성되어 15 주간 진행하였다. 실험군의 의사소통 능력에 대한 사전-사후조사는 설문지로 조사하였으며 수업 경험은 실험군에게 설문지로 조사 하였다. 자료분석은 SPSS 20.0 프로그램을 이용하여 분석하였다. 연구결과 임상 실무 기반 간호 시뮬레이션 교육 후 의사소통 능력의 점수는 실험군 164.5 점으로 대조군 156.8 점보다

Received: June 02, 2021; $1^{\text {st }}$ Review Result: July 17, 2021; $2^{\text {nd }}$ Review Result: September 01, 2021 Accepted: October 31, 2021 
높아 통계적으로 유의하였으며 $(\mathrm{t}=4.33, \mathrm{p}<.001)$, 시뮬레이션 교육을 받은 실험군의 간호 시 뮬레이션 수업 경험의 점수도 통계적으로 유의하였다 $(\mathrm{t}=15.38, \mathrm{p}<.001)$. 본 연구에서 실시한 간호 시뮬레이션 교육은 학생들의 의사소통 능력을 향상하며, 수업에 대한 긍정적인 경험을 하였다고 판단된다. 이에 대상자의 건강 문제를 해결하기 위하여 다양한 분야에서 적극적인 시뮬레이션 교육을 실시하여야 할 것이며, 시뮬레이션 교육은 실습을 시작하는 학년에서부터 실시하여 현장 임상 실습 수행 능력을 증진시킬 수 있을 것으로 보인다.

핵심어: 시뮬레이션, 임상 실무, 의사소통 능력, 수업 경험

\section{1. 서론}

간호 교육은 이론교육과 실습교육으로 이루어지고 있는데 이론교육은 대부분 강의로 진행되는 교육 방법으로 지식 전달면에서는 효과적인 교육방법이지만 간호 현장에서 이론적 기반으로 간호업무 능력을 향상하는 교육방법으로 제한이 있을 것이다. 현재 간호역량을 증진하기 위한 실습교육은 간호에 대한 이론을 학습하고 이렇게 학습된 지식을 실무에 실제로 통합하고 적용하여 대상자의 건강문제를 해결할 수 있는 능력을 키우고 적용하여 간호역량을 증진시키기 위해서 필수적인 교육방법이다[1].

실제 간호 현장에서 다양한 대상자의 건강 문제를 해결하기 위한 간호지식, 간호 수행방법 및 여러 상황에 직면할 경우 필요한 대처능력을 갖춘 간호사를 양성하고 현장에서 실시하는 임상 실습을 보완하기 위하여 강의실에서 학습한 이론과 임상 실습에서 배운 실제를 통합하고 적용할 수 있도록 다양한 상황을 구축하여 실시하는 간호 시뮬레이션 교육 방법을 시도해야 한다[2].

시뮬레이션이란 멀티미디어 기술, 모형, 인체모형 시뮬레이터 등을 이용하여 재현된 임상 환경에서 학생이 사람과 사물을 통해 상호작용하고 문제나 상황을 해결하기 위해 재현된 임상 상황에 따라 의사 결정하는 것으로 이전의 경험과 배운 지식을 적용하고 그 분야에 대하여 피드백을 받는 실제적인 현장 체험 학습이다[3]. 시뮬레이션은 실제와 유사한 다양하고 광범위한 상황이 놓인 조건들이 통합되어 학습자들은 이를 바탕으로 임상의 문제를 추론하도록 하며, 즉각적인 피드백을 통해 잘못된 행동을 교정하고 임상 상황에서 즉적으로 평가할 수 없거나 어려운 임상 문제를 해결하는 수행 정도를 평가 할 수 있다고 하였다[4].

간호 교육에서 시뮬레이션 교육을 적용한 선행연구를 살펴보면 신규간호사 교육에서는 비판적 사고능력, 문제해결 과정[5], 임상 의사결정 능력의 향상에 효과를 보였으며[6], 간호학생을 대상으로 시뮬레이션 기반 교육을 시행하고 지식, 임상 수행능력, 문제해결 과정에 효과가 있음을 보고하였다[7].

효과적인 의사소통 능력은 간호 교육의 목표 중 하나로 많은 간호 교육에서 실시하는 학습성과 달성에 포함되는 항목으로 전문직 간호사가 갖추어야 할 가장 중요한 능력이므로 이를 증진하기 위하여 시뮬레이션을 적용한 교육을 통하여 의사소통 능력을 실무에서 적용하여 임상 실무수행 역량을 증진한다고 하였다[8].

간호 시뮬레이션교육은 최적의 구조와 시스템을 구축하고 현장 상황에 대비한 지속적인 교육을 실시하여 실수를 통해 배우는 단계로 임상 실습을 통해 이론과 실습을 병행해야 하는 간호학생이 대상자의 문제를 포괄적으로 접근할 수 있는 역량을 
강화하도록[9] 임상 실무를 기반으로 한 모듈을 개발하고 시뮬레이션 교육을 수행하여 임상판단 수행력과 의사소통 능력을 향상시킬 수 있을 것이다. 또한 시뮬레이션 교육에 대한 부담감이나 부정적인 정서를[10][11] 파악하여 임상 실습시 간호학생으로 수행해야 하는 간호 수행에 대하여 자신감 있게 수행하도록 시뮬레이션 교육에 참여하는 학생들에게 긍정적인 수업을 경험할 수 있도록 해야 할 것이다.

이에 본 연구는 임상 실무 기반 간호 시뮬레이션 교육에 참여하는 학생들이 임상적 간호 수행능력을 증진시키기 위하여 대상자의 건강 문제를 해결하기 위한 효과적인 의사소통 능력을 향상시키는 경험을 할 수 있는 교육을 경험하도록 모듈을 개발하여 이를 적용하여 의사소통 능력에 미치는 영향을 알아보기 위함이며 구체적인 목적은 다음과 같다.

첫째, 대상자의 특성을 파악하고 동질성을 검정한다.

둘째, 임상 실무 기반 간호 시뮬레이션 교육이 의사소통 능력에 미치는 효과를 분석한다.

셋째, 임상 실무 기반 간호 시뮬레이션 교육이 실험군의 수업 경험에 미치는 효과를 분석한다.

\section{2. 연구방법}

\section{1 연구 설계}

본 연구는 임상 실무 기반 간호 시뮬레이션 교육(이하, 간호 시뮬레이션 교육)을 간호학생에게 제공하여 의사소통 능력 및 수업 경험의 효과를 파악한 비동등성 대조군 사후 유사실험 연구이다.

\section{2 연구대상}

본 연구의 대상자는 $\mathrm{K}$ 시와 $\mathrm{J}$ 도에 위치한 각 1 개 대학에 재학 중인 3 학년 간호학생으로 실험군, 대조군 모두 간호 시뮬레이션 교육과정을 이수하거나 경험하지 않았다. 하나의 대학은 시뮬레이션 수업을 수강하는 학생으로 실험군 122 명, 또 하나의 대학은 시뮬레이션 수업을 수강하지 않는 학생으로 대조군 132 명 총 254명이다.

본 연구의 목적을 이해하며 연구에 참여는 동의서에 서면 동의한 자로 정규교육과정 내에서 실시하는 연구이지만 연구의 참여나 중도탈락, 연구 결과가 학점에 영향을 미치지 않으며 수집된 자료는 익명성이 보장되며 연구의 목적으로만 사용함을 충분히 설명하였다. 본 연구의 대상자 수를 결정하기 위하여 G*Power 프로그램에 의해 양측검정으로 유의수준 .05, 효과 크기 0.5에서 검정력 .95를 유지하기 위한 표본수는 실험군 108명, 대조군 108 명 총 216명을 근거로 표본 수를 산출하였다.

\section{3 연구 도구}

\subsection{1 의사소통 능력}

의사소통이란 서로의 인정된 목표나 방향을 향해 생각, 느낌 또는 아이디어를 교환하고 발전시키는 양방향 과정으로 파악될 수 있다. 본 연구의 의사소통 능력은 '대인 간 의사소통 능력 모델' 을 토대로 개발한 대학생 및 성인의 생애 능력 중 
의사소통 능력을 측정하기 위한 도구로 5 개의 하위요소인 해석 능력 14 문항, 역할수행 능력 14 문항, 자기 제시 능력 7 문항, 목표설정 능력 7문항, 메시지 전환 능력 7 문항으로 구성된 총 49문항, 5점 척도의 도구이다[12]. 도구개발 당시 신뢰도는 Cronbachs' alpha= .85이었으며, 본 연구에서 도구의 신뢰도는 Cronbachs' alpha= .83이었다.

\subsection{2 수업 경험}

수업 경험이란 수업 과정에서 학습자들이 느끼는 경험의 질을 의하며 본 연구에서 수업 경험은 경험 표 집양식과 수업 만족도로 구성되었다. 경험 표집 양식은 선행연구의 도구를 사용하여[13] 본 연구에 맞게 수정 보완하여 인지적 영역, 정서적 영역, 동기적 영역의 3 영역, 총 25 문항으로 구성되어 있다. 경험적 표집 방식에 해당하는 1 번 문항은 개방형 질문으로 구성되어 서술하도록 하였다. 2 번 문항에서 16 번 문항은 10 점 척도로 0점에서 135점, 17 번 문항에서 25문항은 7점 척도로 9점에서 63점의 범위를 나타내었고 수업 만족도는 1점에서 5점의 범위를 나타낸다. 따라서 수업 경험은 총 10 점에서 203점의 범위로 점수가 높을수록 긍정적인 수업 경험을 나타낸다. 선행연구에서 Cronbachs' alpha= .93이었으며[13], 본 연구에서 도구의 신뢰도는 Cronbachs' alpha $=.89$ 이었다.

\section{4 자료수집 및 방법}

본 연구의 자료 수집을 위하여 간호 시뮬레이션 교육에 대하여 목적과 진행 절차를 충분히 설명하고 참여하기로 서면 동의한 자로 정규교육과정 내에서 실시하는 연구이지만 익명성이 보장되며, 연구의 참여하거나나 중도탈락한 결과가 수업 평가에 전혀 영향을 미치지 않음을 충분히 설명하였다. 사전 자료 수집은 감염병 예방 수칙을 충실히 준수하여 수집하였다. 실험군, 대조군 모두 간호 시뮬레이션 교육 시작 전 2021년 3월 첫주동안 실시하였고 사후 자료 수집은 15주간의 임상 실무 기반 간호 시뮬레이션 교육 종료 후 교육을 받은 실험군은 2021년 6월 15일, 교육을 받지 않은 대조군은 2021년 6월 마지막주 기간 동안 실시하였다.

실험군의 사전과 사후 자료 수집은 설문지를 통하여 간호 시뮬레이션 교육에 대한 의사소통 능력을 조사하였으며, 실험군의 간호 시뮬레이션 교육에 대한 수업 경험은 설문지를 통하여 사후 조사를 실시하였다.

\section{5 연구 진행 절차}

임상 실무 기반 간호 시뮬레이션 교육은 3 학년 간호학생이 현장실습 시 대상자의 건강문제를 해결하기 위한 효과적인 의사소통 능력의 함양과 간호학생의 임상실습 능력을 향상시키기 위함으로 실험군 122 명에게 교육을 실시하였다. 학습모듈은 본 연구의 대상자들의 임상 실습 학점이 가장 많은 성인 간호학의 실습영역인 수술, 내분비계, 순환기계, 비뇨기계 환자 간호인 4가지 모듈로 구성하였다.

간호 시뮬레이션 교육은 1학점으로 주당 2시간 15주 수업으로 모듈당 2주씩 시행하였다. 구성된 학습 모듈의 내용은 한국간호교육 평가원에서 제시한 핵심 기본간호술 20 가지 영역이 일부 포함되도록 구성하였다. 내용 타당도와 적절성은 경력 10 년 이상의 성인간호학 교수 2 인과 다양한 분야에서 수업을 실시한 경험이 있는 교수 
1 인이 검토하고 수정 보완하였다.

각 모듈은 사전 학습, 학습 목표, 환자 정보, 의사 처방 및 검사 결과, 학습평가 체크리스트, 자가평가 체크리스트, 실습실 체크리스트로 구성되었으며, 평가자 간 일관성이 유지되도록 평가자 4명이 훈련을 통하여 타당성 있고 일관성 있도록 평가를 실시하였다. 사전학습 10분, 시뮬레이션 교육 50분, Debriefing 40분 총 100분으로 시률레이터와 OSCE Room을 활용하여 팀으로 실습하였고 팀원은 3 4명으로 구성하였다. 리더와 팀원으로 구성하여 매주 팀원 내의 역할을 바꾸어가며 팀 리더와 팀원이 해야 할 주요한 역할을 배분하여 모듈마다 필요한 간호를 수행하도록 하였다.

사전학습은 시뮬레이션 수업을 담당한 교수 2 인이 개발한 교재를 통하여 각 모듈에서 요구되는 사전학습 내용을 숙지하고 시뮬레이션 교육에 참여하도록 하였다. 시뮬레이션 교육은 SimMan 시뮬레이터를 이용하여 간호를 수행하고 교수는 Control Room에서 모니터 및 실습실 상황을 실시간 녹화하였고 필요하면 학생들에게 조언해 주었다.

Debriefing은 시뮬레이션 교육 후 학생들과 함께 수업내용에 대한 평가를 3 단계에 걸쳐 실시하였다. 또한 팀별로 수행한 간호를 Mapping을 통하여 자유롭게 표현하도록 하여 조별로 의견을 나누고 상호보완점을 인지할 수 있는 정보를 공유하도록 하였으며 수업 경험에 대해 조별 및 개별 발표하여 수업 내용에 대한 피드백을 실시 후 자가 평가함으로써 수업을 마무리하였다[표 1].

\section{6 자료 분석 방법}

수집된 자료는 SPSS 20.0을 이용하여 전산통계 처리하였다. 연구 대상자의 일반적 특성 등은 실수와 백분율, 평균과 표준편차로 구하였으며, 임상 실무 기반 간호 시률레이션 교육이 대상자의 의사소통 능력에 미치는 효과는 t-test, ANOVA, 간호 시률레이션 교육이 실험군의 수업 경험에 미치는 효과는 paired t-test로 분석하였다. 측정 도구의 신뢰도는 Cronbach's alpha로 검정하였다.

[표 1] 시뮬레이션 교육 과정

[Table 1] Simulation Education Process

\begin{tabular}{|c|c|c|}
\hline $\begin{array}{l}\text { Simulation } \\
\text { Process }\end{array}$ & Time & Content \\
\hline $\begin{array}{c}\text { Prior } \\
\text { Learning }\end{array}$ & 10 & $\begin{array}{l}\text { Primary care patients with each module, Identify and nursing practice each module, } \\
\text { Nursing diagnoses each module }\end{array}$ \\
\hline & & $\boldsymbol{\nabla}$ \\
\hline $\begin{array}{l}\text { Simulation } \\
\text { Education }\end{array}$ & 60 & Case summary introduction patients and situations each module \\
\hline & & $\nabla$ \\
\hline \multirow{9}{*}{ Debriefing } & \multirow{9}{*}{40} & Analysis phase \\
\hline & & $\boldsymbol{\nabla}$ \\
\hline & & Application phase \\
\hline & & $\boldsymbol{\nabla}$ \\
\hline & & Description phase \\
\hline & & $\boldsymbol{\nabla}$ \\
\hline & & Mapping \\
\hline & & $\boldsymbol{\nabla}$ \\
\hline & & Feedback \& Self Evaluation \\
\hline
\end{tabular}




\section{3. 연구 결과}

\section{1 연구 대상자의 일반적 특성에 대한 동질성 검사}

본 연구 대상자의 일반적 특성으로 연령은 22 23세가 $44.9 \%$ 이었으며 실험군과 대조군 간의 일반적 특성에 대한 동질성 검정은 통계적으로 유의한 차이가 없었다[표 2]. 또한 대상자의 의사소통 능력은 실험군 147.7점, 대조군 149.4점으로 통계적으로 유의한 차이가 없어 동질한 집단으로 나타났다[표 3].

\section{[표 2] 연구대상자의 일반적 특성}

[Table 2] Homogeneity of Characteristics of the Subjects

\begin{tabular}{|c|c|c|c|c|c|c|c|}
\hline \multirow{2}{*}{ Variables } & \multirow{2}{*}{ Category } & \multicolumn{2}{|c|}{$\begin{array}{c}\text { Exp. } \\
(\mathrm{n}=122)\end{array}$} & \multicolumn{2}{|c|}{$\begin{array}{c}\text { Cont. } \\
(\mathrm{n}=132)\end{array}$} & \multirow[t]{2}{*}{$\mathrm{t} / \mathrm{F}$} & \multirow{2}{*}{$p$} \\
\hline & & $\mathrm{n}$ & $\%$ & $\mathrm{n}$ & $\%$ & & \\
\hline \multirow{2}{*}{ Gender } & Male & 15 & 12.3 & 12 & 9.1 & \multirow{2}{*}{-.82} & \multirow{2}{*}{.412} \\
\hline & Female & 107 & 87.7 & 120 & 90.9 & & \\
\hline \multirow{3}{*}{ Age(yr) } & 20 below & 35 & 28.7 & 40 & 30.3 & \multirow{3}{*}{-.03} & \multirow{3}{*}{.973} \\
\hline & 21 & 57 & 46.7 & 57 & 43.2 & & \\
\hline & 22 over & 30 & 24.6 & 35 & 26.5 & & \\
\hline \multirow{4}{*}{$\begin{array}{l}\text { Entered school } \\
\text { motivation }\end{array}$} & Own will & 40 & 32.8 & 49 & 37.1 & \multirow{4}{*}{-1.28} & \multirow{4}{*}{.201} \\
\hline & Family advice & 50 & 41.0 & 31 & 23.5 & & \\
\hline & Employment & 40 & 32.8 & 43 & 32.6 & & \\
\hline & Attractive career & 22 & 18.0 & 9 & 6.8 & & \\
\hline \multirow{3}{*}{$\begin{array}{l}\text { Major } \\
\text { difficult }\end{array}$} & hool work \& practice & 83 & 68.0 & 100 & 75.8 & \multirow{3}{*}{-1.25} & \multirow{3}{*}{.210} \\
\hline & Relationships with classmates & 18 & 14.8 & 27 & 20.5 & & \\
\hline & Other & 21 & 17.2 & 5 & 3.8 & & \\
\hline \multirow{3}{*}{$\begin{array}{l}\text { Information } \\
\text { of simulation }\end{array}$} & Know & 2 & 1.6 & 2 & 1.5 & \multirow{3}{*}{-1.64} & \multirow{3}{*}{.102} \\
\hline & Know a little bit & 84 & 68.9 & 85 & 64.4 & & \\
\hline & Do not know & 36 & 29.5 & 45 & 34.1 & & \\
\hline \multirow{2}{*}{$\begin{array}{c}\text { Team learning } \\
\text { experience }\end{array}$} & Yes & 44 & 36.1 & 61 & 46.2 & \multirow{2}{*}{-1.65} & \multirow{2}{*}{.101} \\
\hline & No & 78 & 63.9 & 71 & 53.8 & & \\
\hline \multirow{2}{*}{ Position in team } & Team leader & 17 & 13.9 & 19 & 14.4 & \multirow{2}{*}{.10} & \multirow{2}{*}{.917} \\
\hline & Team member & 105 & 86.1 & 113 & 85.6 & & \\
\hline
\end{tabular}

[표 3] 동질성 검정

[Table 3] Homogeneity of Research Variables

\begin{tabular}{|c|c|c|c|c|}
\hline \multirow{2}{*}{ Variables } & $\begin{array}{c}\text { Exp. } \\
(\mathrm{n}=122)\end{array}$ & $\begin{array}{c}\text { Cont. } \\
(\mathrm{n}=132)\end{array}$ & \multirow{2}{*}{$\mathrm{t}$} \\
\cline { 2 - 5 } & Mean $\pm \mathrm{SD}$ & Mean $\pm \mathrm{SD}$ & 1.16 & .136 \\
\hline Communication Ability & $147.7 \pm 4.4$ & $149.4 \pm 4.4$ & \\
\hline
\end{tabular}




\section{2 임상 실무 기반 간호 시뮬레이션 교육이 연구 대상자의 의사소통 능력에 미치는 효과}

임상 실무 기반 간호 시뮬레이션 교육 후 실험군의 의사소통 능력의 점수는 총점 164.5점으로 대조군 156.8점보다 높아 통계적으로 유의하였으며 $(\mathrm{t}=4.33, \mathrm{p}<.001)$, 하부 영역으로 해석 능력 $(\mathrm{t}=5.13, \mathrm{p}<.001)$, 역할수행 능력 $(\mathrm{t}=3.66, \quad \mathrm{p}<.001), \quad$ 자기제시 능력 $(\mathrm{t}=-2.31, \mathrm{p}=.021)$, 목표설정 능력 $(\mathrm{t}=3.11, \mathrm{p}=.002)$, 메시지 전환 능력 $(\mathrm{t}=3.15$, $\mathrm{p}=.002)$ 에서 통계적으로 유의하였다[표 4].

[표 4] 시뮬레이션 교육이 의사소통 능력에 미치는 효과

[Table 4] Simulations Education Effects on Communication Ability

\begin{tabular}{|c|c|c|c|c|c|c|}
\hline \multirow{2}{*}{ Variables } & \multicolumn{2}{|c|}{$\begin{array}{c}\text { Exp. } \\
(\mathrm{n}=122)\end{array}$} & \multicolumn{2}{c|}{$\begin{array}{c}\text { Cont. } \\
(\mathrm{n}=132)\end{array}$} & \multirow{2}{*}{$\mathrm{t}$} \\
\cline { 2 - 6 } & Mean & SD & Mean & SD & & \\
\hline Communication ability & 164.5 & 14.3 & 156.8 & 14.0 & 4.33 & $<.001$ \\
\hline Ability to interpret & 24.5 & 2.7 & 22.8 & 2.7 & 5.13 & $<.001$ \\
\hline Ability to perform the role & 25.6 & 3.1 & 24.1 & 3.6 & 3.66 & $<.001$ \\
\hline Self-pre sentation ability & 16.4 & 4.2 & 17.6 & 4.0 & -2.31 & .021 \\
\hline Ability to goals & 23.3 & 2.8 & 22.2 & 2.4 & 3.11 & .002 \\
\hline Massage switchover & 24.9 & 3.7 & 23.4 & 3.6 & 3.15 & .002 \\
\hline
\end{tabular}

\section{3 임상 실무 기반 간호 시뮬레이션 교육이 연구 대상자의 수업 경험에 미치는 효과}

임상 실무 기반 간호 시뮬레이션 교육을 받은 실험군의 수업 경험 점수는 193.0점이었다. 수업 경험의 구성 요소인 수업 만족도는 4.2점, 수업 중 경험은 94.4점, 수업 중 느낌은 52.4점으로 모두 통계적으로 유의하였다 $(\mathrm{t}=15.38, \mathrm{p}<.001)$ [표 5].

[표 5] 간호 시뮬레이션 교육이 실험군의 수업 경험에 미치는 효과

[Table 5] Simulations Education Effects on Training Experience in Experimental Groups

\begin{tabular}{|c|c|c|c|c|}
\hline \multirow{2}{*}{ Variables } & \multicolumn{2}{|c|}{$\begin{array}{c}\text { Exp. } \\
(\mathrm{n}=122)\end{array}$} & $\mathrm{v}$ \\
\cline { 2 - 4 } & Mean & SD & & $<.001$ \\
\hline Simulation training on teaching experience & 193.0 & 75.5 & 15.38 & $<.001$ \\
\hline Satisfaction & 4.2 & 0.7 & 70.21 & $<.001$ \\
\hline During teaching experience & 94.4 & 12.4 & 83.82 & $<.001$ \\
\hline
\end{tabular}




\section{4. 결론}

본 연구는 일 대학에 재학 중인 3학년 간호학생을 대상으로 임상 실무 기반 간호 시뮬레이션 교육을 적용함으로써 간호 교육을 통하여 성취해야 할 중요한 학습성과인 의사소통 능력을 향상시키고 긍정적인 수업 경험을 끌어내기 위하여 시도되었다.

간호 시뮬레이션 교육은 간호학생들의 간호학적 지식과 기술을 연계시키며 통합적인 사고를 통합함으로써 의사소통 능력을 증진시키는데 효과적인 교육 방법이다. 본 연구의 결과 간호 시뮬레이션 교육 후 실험군의 의사소통이 대조군보다 향상된 것으로 나타났다. 인간을 대상으로 하는 학문인 간호학 전공자들에게 입학과 동시에 효과적인 의사소통 능력이 매우 중요한 과제로 강조되면서 타 전공과 달리 교양과목 등에 효과적인 의사소통과 관련된 교과목을 개설하여 학생들에게 의사소통에 관한 체계적인 학습기회를 제공하고 있지만 교수-학습 방법에 따라 그 성취도는 매우 다를 수 있다[8]. 또한 의사소통능력은 단순한 교과목 수강으로 터득할 수 있는 능력이 아니며, 체계적인 학습과 더불어 생활 속에서 학습한 지식을 적용하고 활용함으로써 터득할 수 있는 것이다[10][14].

간호 시뮬레이션 교육 시 연구자들은 팀원은 3 4명으로 구성하여 간호사 1 , 간호사 2 , 의사, 보호자의 역할을 모든 구성원이 순차적으로 수행하도록 하였다. 간호 시뮬레이션 교육시 팀 리더의 역할과 구성원 각자가 역할별 업무를 효율적으로 수행해야 효과적인 인원을 팀별 4 5인으로[13], 본 연구에서 구성한 팀원의 숫자는 각자의 역할을 충분히 발휘할 수 있도록 적절히 구성되었다고 사료된다. 그러므로 대상자의 문제해결에 대한 성과를 높일 수 있도록 각 팀원 간의 의사소통을 증진시킨 것으로 판단된다.

또한 학생들은 핵가족화로 대화의 상대가 줄었고 학교에서는 물론 가정에서도 의사소통의 기회를 충분히 얻지 못하고 있어 팀 구성의 간호 시률레이션 교육을 통하여 지속적으로 의사소통 능력을 향상시킬 수 있는 방법을 간호 교육에 적용하는 것은 매우 의미 있고[14], 지식을 전달하는 교수-학습 방법보다 팀 구성의 시뮬레이션 교육은 각 구성원의 맡은 역할을 구성하여 스스로 책임감을 느끼고 활발한 토의와 적극적인 의사 참여 활동 및 교육과정이 진행되는 동안 소그룹 내의 구성원들과 팀 리더, 팀원의 역할을 모두 경험할 수 있어 상호작용할 수 있게 한다[14][15].

간호 시뮬레이션을 활용한 교육이 학생 상호 간 의사소통, 전문적인 간호 기술 등에서 유의한 변화를 나타냈다고 하였으며[16], 또한 의사소통 기술 향상, 자신감 및 지식이 향상되었고, 환자 사정, 의사소통 등에서 유의한 향상을 보여[17] 본 연구와 유사한 결과를 나타내었다. 그러므로 간호 시뮬레이션 교육을 받는 동안 의사소통 기술을 사용함으로써 자기 생각과 의견을 충분히 표현하고 팀 구성원들과의 상호작용을 통하여 팀원과의 의사소통하는 능력을 향상시킨 것으로 보인다.

간호 시뮬레이션 적용 후 충분한 debriefing을 통하여 시뮬레이션 기반 학습에 대하여 긍정적인 영향을 줄 수 있음으로[18], 시물레이션 교육에 대한 만족감과 성취감이 향상될 수 있도록 학생 스스로 feedback 하는 과정을 갖도록 control room에서 모니터 및 실습실 상황을 실시간 녹화하였으며 조별 실습 후 학생들은 debriefing을 통하여 수업내용에 대한 평가를 3 단계에 걸쳐 실시하였다. 실습한 간호 수행에 대하여 조별로 의견을 나눔으로써 정보를 공유하였으며 수업 경험에 대해 조별 및 개별 발표함으로써 수업 내용에 대한 피드백을 하여 의사소통이 향상되었다고 사료된다.

본 연구에서 의사소통의 하부영역인 역할수행 능력에서 가장 높은 점수를 
나타내었는데, 소집단 구성원들은 자기가 맡은 역할에 대하여 책임감을 느끼고 능동적으로 의견을 제시하며 수업이 진행되는 동안 집단 구성원들과 팀원의 역할과 팀장의 역할을 고루 수행하며 개방적인 대화를 통하여 상호작용하는 모습이 관찰되었다.

선행연구에서도 학습이 진행되는 동안 실험군의 수업 경험 표집과 수업 만족도를 토의법을 적용한 대조군과 비교한 협동 학습을 적용한 실험군에서 학습자들의 수업 집중도 등의 인지적 효율성이 높았고, 수업 동안 행복하고, 즐겁고, 사교적인 정서 상태, 신나고 활발하게 활동하였으며, 열린 태도로 수업에 적극적으로 참여하였다고 하여[14][18] 본 연구의 결과를 뒷받침하였다.

학생들은 문제해결을 위해 학생들 간에 도움을 주고받고 의견을 주고받는 것이 중요하다. 본 연구에서 시뮬레이션 교육 후 실시한 debriefing과 자유로운 mapping을 통하여 대상자들은 시뮬레이션 수업을 위해 준비한 사전학습은 간호 수행을 하는 데 있어 주요한 기반이라고 인식하였고, 시뮬레이션 수업 후 mapping은 다른 팀원들과 자신이 수행한 간호 수행을 재평가하게 되고 다른 팀과 의견을 교환함으로써 미래의 간호사가 익혀야 할 간호에 대해 간접 경험을 할 수 있어 긍정적인 수업 경험을 끌어낸 것으로 보인다

이에 본 연구의 대상자들은 그룹 내에서 이루어진 상호작용, 상호보완을 통하여 간호 시뮬레이션 교육을 성공적으로 이끌었다고 판단되며 대상자의 건강 문제를 해결하기 위하여 다양한 분야에서 적극적인 간호 시뮬레이션 교육을 실시하여야 할 것이며, 간호 시뮬레이션 교육을 임상 실습을 준비하고자 하는 학년에서부터 실시하여 현장 임상 실습 수행 능력을 강화시킬 수 있을 것이다.

\section{5. 감사의 글}

본 연구는 초당대학교 2021년도 학술연구과제 연구보조비 지원으로 이루어 짐.

\section{References}

[1] P. M. Ironside, A. M. McNelis, P. Ebright, Clinical Education in Nursing: Rethinking Learning in Practice Settings, Nursing Outlook, (2014), Vol.62, No.3, pp.185-191.

[2] M. A. Kelly, P. Hager, R. Gallagher, What Matters Most? Students' Rankings of Simulation Components That Contribute to Clinical Judgement, Journal of Nursing Education, (2014), Vol.53, No.2, pp.97-101.

[3] Woo-sook Lee, Kap-chul Cho, Sun-Hee Yang, Young-Sook Roh, Gyu-Young Lee, Effects of Problem-Based Learning Combined with Simulation on the Basic Nursing Competency of Nursing Students, Journal of Korean Academy of Fundamentals of Nursing, (2009), Vol.1, pp.64-72.

[4] Hye-kyung Oh, Young-in Han, Effects of Nursing Simulation-Based Education on Problem Solving Process and SelfEfficacy of Nursing College Students, Journal of Korean Health Service Management, (2012), Vol.6, No.4, pp.245254.

[5] A. D. Ackermann, G. Kenny, C. Walker, Simulator Programs for New Nurses' Orientation, Journal of Nurses in Staff Development, (2007), Vol.23, No.3, pp.136-139, DOI: 10.1097/01.NND.0000277183.32582.43

[6] Hye-kyung Heo, Young-sook Noh, Effects of a Simulation Based Clinical Reasoning Practice Program on Clinical Competence in Nursing Students, Korean Journal of Adult Nursing, (2013), Vol.25, No.5, pp.574-584, UCI: G704000678.2013.25.5.006

[7] Jin-ju Yang, Development and Evaluation of a Simulation-Based Education Course for Nursing Students, Journal of 
Korea Society of Adult Nursing, (2008), Vol.20, No.4, pp.548-560, UCI: G704-000678.2008.20.4.005

[8] Ga-Eul Joo, Kyeong-Yae Sohng, Hee-Ju Kim, Effects of a Standardized Patient Simulation Program for Nursing Students on Nursing Competence, Communication Skill, Self-efficacy and Critical Thinking Ability for Blood Transfusion, The Journal of Fundamentals of Nursing, (2015), Vol. 22, No.1, pp.49-58, DOI: 10.7739/jkafn.2015.22.1.49

[9] S. E. Chung, S. H. Lee, Nursing Student"s Experience of Education using Simulation, Journal of Qualitative Research, (2010), Vol.11, No.1, pp.50-59.

[10] M. Akaike, M. Fukutomi, M. Nagamune, A. Fujimoto, A. Tsuji, K. Ishida, T. Iwata, Simulation-Based Medical Education in Clinical Skills Laboratory, The Journal of Medical Investigation, (2012), Vol.59, No.1-2, pp.28-35, DOI: $10.2152 /$ jmi. 59.28

[11] Hee-Young Kang, Eun-Young Choi, Hae-Ran Kim, Nursing Student's Experiences in Team Based Simulation Learning, The Journal of Korean Academic Society of Nursing Education, (2013), Vol.19, No.1, pp.5-15, DOI: 10.5977/jkasne.2013.19.1.5

[12] S. J. Lee, Y. K. Jang, H. A. Lee, K. E. Park, Study on the Development of Life-Skills : Communication, Problem Solving, and Self-Directed Learning, Korean Educational Development Institute, (2003), https://www.dbpia.co.kr/journal/articleDetail?nodeId=NODE09358383

[13] Oon-hee Yee, Ju-young Ha, Young-mi Ahn, The Influences of Pair Discussion on Nursing Students' Learning Experience in Problem-Based Learning: a Qualitative Inquiry, International Journal of Advanced Nursing Education and Research, (2018), Vol.3, No.1, pp.105-110, DOI: 10.21742/ijaner.2018.3.1.18

[14] Jeong-Hye Park, Effectiveness of Cooperative Learning on Nursing Students' Performance and Experience, The Journal of Korean Academic Society of Nursing Education, (2010), Vol.16, No.2, pp.202-212, DOI: 10.5977/JKASNE.2010.16.2.202

[15] Mi-Won Kim, Hee-Joo Lee, Improvement of Verbal and Nonverbal Communication Skills of Nursing Students through Self-Objectification, International Journal of Advanced Nursing Education and Research, (2018), Vol.3. No.1, pp.31-36, DOI: 10.21742/ijaner.2018.3.1.06

[16] Yeon ja Kim, You jung Kim, An Analysis on the Recognition of Life after Death in Nursing College Students Who Experienced Clinical Training, International Journal of Advanced Nursing Education and Research, (2017), Vol.2, No.1, pp.183-188, DOI:10.21742/IJANER.2017.2.1.31

[17] Mi-Young Moon, Young-Ok Lee, Performance of Therapeutic Communication Knowledge Application and Technique During Clinical Training of Nursing College Students, International Journal of Advanced Nursing Education and Research, (2019), Vol.4, No.3, pp.19-24, DOI: 10.21742/ijaner.2019.4.3.04

[18] Mi-hyun Jeong, Yo-han Seo, The Effect of Debriefing in Nursing Simulation based Education on the Vocational Basic Competencies, Journal of the Korean Applied Science and Technology, (2018), Vol.35, No.1, pp.20-35, DOI: 10.12925/jkocs.2018.35.1.20 